\title{
Improving SSVEP-BCI Performance Using Pre-Trial Normalization Methods
}

\author{
James Henshaw \\ Department of Electronic and \\ Electrical Engineering \\ University of Sheffield \\ Sheffield, England \\ Email: jhenshaw1@ sheffield.ac.uk
}

\author{
Wei Liu \\ Department of Electronic and \\ Electrical Engineering \\ University of Sheffield \\ Sheffield, England \\ Email:w.liu@sheffield.ac.uk
}

\author{
Daniela M. Romano \\ Sheffield Robotics \\ University of Sheffield \\ Sheffield, England \\ Email: d.m.romano@sheffield.ac.uk
}

\begin{abstract}
A brain-computer interface (BCI) enables users to communicate through a computer using only their brain signals, by extracting brain signal features containing information representative of the user's intent, and can be used in a wide variety of areas such as entertainment, rehabilitation, or assistive technologies. In this paper, two novel normalization methods are assessed with the aim of improving the quality of the extracted features: Baseline-Corrected canonical correlation analysis (BCCCA), and Scaled CCA. Both methods are found to be able to improve classification accuracy in conditions using frequencies with a large range, whilst $\mathrm{BC}-\mathrm{CCA}$ is the superior of the two, improving SSVEP detection accuracy by as much as $\mathbf{9 . 2 2 \%}$.
\end{abstract}

Keywords-brain-computer interface, BCI, SSVEP, normalization, EEG.

\section{INTRODUCTION}

A brain-computer interface (BCI) is operated using braingenerated information, thereby providing the user with an alternative communication or control channel that does not require the brain's normal peripheral nerve and muscle pathways [1]. Operating a BCI is a multi-step process [2], which involves acquiring a signal from the user's brain, preprocessing it to reduce artifacts, extracting useful features that can be used to make inferences about the user's current cognitive state, and then translating these features into a form that can be used to communicate with an external device, and finally, providing feedback so the user can see that the BCI is working (Fig. 1) and adjust their approach if necessary. Overall, BCIs can be viewed as an inter-cognitive communication system [3], which transfers the brain's neural signals into an alternative format, providing the user with the ability to communicate directly with external devices. In the last few years, BCI has begun to find its place within the field of Cognitive Infocommunications (CogInfoCom), including studies into using brainwaves to operate a mobile robot [4], and detecting a user's mental arousal levels using a BCI [5].

There are several methods for operating a BCI [6]; one of the most popular involves exploiting the brain's steadystate visually evoked potential (SSVEP [7]) response. SSVEPs are a phase-locked brain response triggered by fixing the user's gaze upon a repetitive visual stimulus (RVS) such as a flashing light [8], or a reversing pattern [9]. The SSVEP is produced by groups of neurons which output a repetitive signal matching the RVS frequency, maintained for the duration of the fixation period. Correctly identifying the user's SSVEP frequency allows a correct command to be sent to the BCI. The SSVEP response can be detected by recording brain activity from electrodes placed around the occipital and parietal lobes [10], and interpreting the brain signal using classification algorithms. SSVEP-BCIs are popular due to their short training time, high classification rate [11], and the fact that they can be detected using non-invasive neuroimaging methods such as electroencephalography (EEG). They have been used in a diverse range of applications, including BCI-controlled computer games [12], exoskeletons [13], wheelchairs [14], [15], and robotic humanoids [16].

Currently, two of the most popular methods for SSVEP feature extraction are: canonical correlation analysis (CCA, [17], [18], [19], [20], [21]), which calculates the correlation between the user's EEG signal and the target frequencies, and power spectral density analysis (PSDA, [22], [23]), which uses frequency components of the EEG signal as features for classification. CCA has a high accuracy and does not need training data. One problematic characteristic of EEG is that the EEG frequency components from low frequencies tend to have higher power than those from high frequencies, making them easier to detect, and leaving a BCI naturally skewed in favour of low frequency SSVEPs. One way to minimize this bias is to only use stimulus frequencies from the same range. However, it would be preferable to adjust our feature extraction methods in a way that gives a balanced result. Having access to more stimulus frequencies means more unique commands can be sent, thus providing a higher information transfer rate. It is noted in [24] that uneven distribution between classification accuracy of classes leads to a skewed performance, whereas the ideal BCI will have an equal chance of selecting any command. This skewness can be alleviated by normalization, also known as feature scaling, which standardizes features based on some relationship within or between feature groups, and reduces the impact of extreme values and/or the difference between features of different classes.

The goal of this research is to improve the classification accuracy of SSVEP-BCIs by normalizing the extracted features from different classes, thereby increasing the chances of high frequency SSVEP responses being correctly identified. Specifically, we compare two novel methods aimed at improving the BCI's ability to send information by improving the quality of the features produced during the feature extraction process. 
We call these methods Baseline-Corrected CCA and ScaledCCA. There have been numerous papers exploring different aspects of the BCI process; however, to the authors' knowledge there is currently very little research into SSVEP normalization methods.

\section{PREVIOUS WORK}

A number of studies have used methods of normalizing EEG signals for SSVEP detection. Nakanishi and colleagues [19] took CCA features from their target frequencies and normalized them against CCA features from neighbouring frequency bands, to help compensate for poorer classification with higher frequency RVS. They found that these features could perform as well as (and sometimes outperform) the standard $\mathrm{CCA}$, and performance improved as the number of neighbours increased. Castillo et al. [22] applied a similar method of normalizing features against neighbouring frequencies using PSDA, where they would normalize against a single value to find the largest ratio. This led to a more accurate BCI and had less variance than the PSDA. Despite a relatively low SNR of the high-frequency visual input, Sakurada et al. [25] created a high frequency SSVEP-BCI with good three-class classification accuracy, normalizing all the RVS frequencies against the inter-trial average of spectral power across the fixation period, and also against competing frequencies. In effect each normalized SSVEP amplitude was the baseline corrected amplitude with the mean amplitude of the (baselinecorrected) competitors subtracted from it. Diez et al. [26] had participants operate a BCI-controlled navigation robot using SSVEP features from high-frequency $(f>35 \mathrm{~Hz})$ RVS. These features were normalized against the periodogram of baseline data collected prior to the study. There was no direct comparison with other normalization methods as this was a navigation study. However, all participants were able to successfully operate the BCI using the baseline-corrected features.

The previous literature illustrates that there are a variety of different ways to improve SSVEP performance using normalization techniques. However, the majority of studies focus on PSDA-based techniques, whereas the current state-of-theart SSVEP-BCI algorithms use CCA and CCA-based methods. Previous research has indicated that it is possible to improve CCA performance using normalization methods, and also that data from the pre-fixation period can be used to normalize the SSVEP response across frequencies, albeit with PSDA. Therefore, based on previous research and the characteristics of EEG, we hypothesize that: (1) SSVEP-BCI performance can be improved by using CCA data from the pre-fixation period, and that; (2) normalization techniques will cause the greatest improvement for classifying RVS frequencies that have a wide range between them.

\section{Methodology}

\section{A. Participants}

Participants were 17 healthy students recruited using the university mailing system (4 female, 13 male) with a mean age of 26.5 years old.

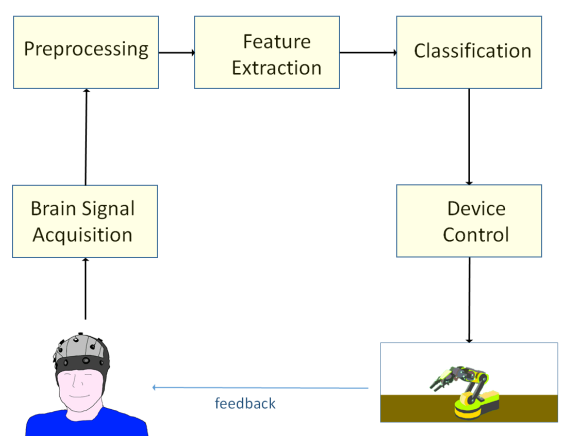

Fig. 1. BCI block diagram

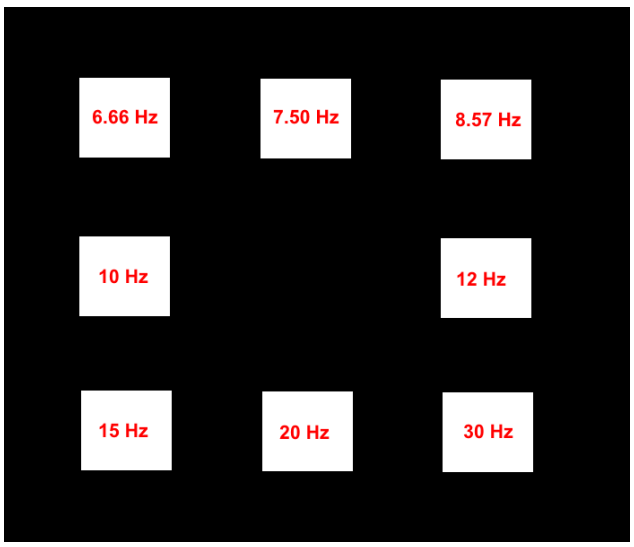

Fig. 2. SSVEP stimulus screen layout

\section{B. SSVEP Stimulus}

An RVS was created and displayed on a separate computer, using code written on MATLAB (MathWorks Inc.) plugin Psychtoolbox ( [27], [28], [29]. Eight SSVEP stimulus frequencies: $6.66,7.5,8.57,10,12,15,20$, and $30 \mathrm{~Hz}$, were produced using the method outlined by Cecotti et al. [30], and displayed on a $60 \mathrm{~Hz}$ screen in a 3x3 layout, as shown in Fig. 2. These are the only RVS frequencies that can be reliably displayed on a $60 \mathrm{~Hz}$ monitor using this method.

\section{Data Collection}

Each participant's EEG activity was recorded as they gazed at the on-screen stimulus, using a Neuroelectrics ${ }^{1}$ Enobio 20-channel EEG system with $\mathrm{AgCl}$ electrodes, referenced to the right mastoid. In a single group of eight trials, the participant was instructed (via the fixation cross) to gaze at subsequent stimulus squares in a left-to-right, top-to-bottom fashion, meaning the frequency values increased for each of the eight trials. This pattern was repeated for all 30 groups of trials, giving a total of 240 trials, which took 30 minutes per participant. Each individual trial lasted seven seconds: a twosecond fixation period, followed by five seconds of SSVEP stimulation (Fig. 3). Participants were given a one-minute break every nine minutes. During recording, participants were seated $60 \mathrm{~cm}$ away from the screen, in a room with reduced natural light.

\footnotetext{
${ }^{1}$ Www.neuroelectrics.com
} 


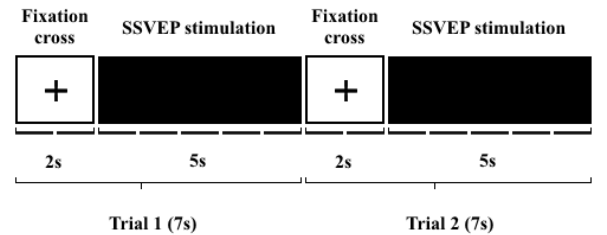

Fig. 3. Trial outline

\section{Standard CCA Feature Extraction}

CCA is a method for identifying the underlying correlation between two multidimensional variables, and has been successfully used to perform unsupervised SSVEP detection [17], [18]. For two multidimensional variables $\boldsymbol{X}$ and $\boldsymbol{Y}$ with weighted linear combinations $\boldsymbol{x}=\boldsymbol{X}^{T} \boldsymbol{W}_{\boldsymbol{X}}$ and $\boldsymbol{y}=\boldsymbol{Y}^{T} \boldsymbol{W}_{\boldsymbol{Y}}$, CCA works by finding the weight vectors $\boldsymbol{W}_{\boldsymbol{X}}$ and $\boldsymbol{W}_{\boldsymbol{Y}}$ which maximise the correlation between $\boldsymbol{x}$ and $\boldsymbol{y}$. This is accomplished by solving the following optimisation problem:

$$
\begin{aligned}
\max _{\boldsymbol{W}_{\boldsymbol{X}}, \boldsymbol{W}_{\boldsymbol{Y}}} \rho(\boldsymbol{x}, \boldsymbol{y}) & =\frac{E[\boldsymbol{x} \boldsymbol{y}]}{\sqrt{E[\boldsymbol{x} \boldsymbol{x}] E[\boldsymbol{y} \boldsymbol{y}]}} \\
& =\frac{E\left[\boldsymbol{W}_{\boldsymbol{X}}^{T} \boldsymbol{X} \boldsymbol{Y}^{T} \boldsymbol{W}_{\boldsymbol{Y}}\right]}{\sqrt{E\left[\boldsymbol{W}_{\boldsymbol{X}}^{T} \boldsymbol{X} \boldsymbol{X}^{T} \boldsymbol{W}_{\boldsymbol{X}}\right] E\left[\boldsymbol{W}_{\boldsymbol{Y}}^{T} \boldsymbol{Y} \boldsymbol{Y}^{T} \boldsymbol{W}_{\boldsymbol{Y}}\right]}},
\end{aligned}
$$

where $E[x]$ represents the expected value of $x$, and $\rho$ is the correlation value, which is maximised with respect to weight vectors $\boldsymbol{W}_{\boldsymbol{X}}$ and $\boldsymbol{W}_{\boldsymbol{Y}}$, thereby calculating the canonical correlation between $\boldsymbol{X}$ and $\boldsymbol{Y}$.

In the case of SSVEP detection, $\boldsymbol{X} \in R^{C \times S}$ is a multidimensional EEG signal with $C$ channels and $S$ samples. $\boldsymbol{Y}_{f} \in R^{2 N_{h} \times S}$ is a multidimensional set of reference signals based on stimulus frequency $f$, with $2 N_{h}$ individual sine waves and $S$ samples, where $N_{h}$ is the number of harmonics. The sine waves are assembled into a matrix [17]:

$$
Y_{f}=\left[\begin{array}{c}
\sin (2 \pi f t) \\
\cos (2 \pi f t) \\
\ldots \\
\sin \left(2 \pi N_{h} f t\right) \\
\cos \left(2 \pi N_{h} f t\right)
\end{array}\right],
$$

where $t$ is the time in seconds. By performing CCA on $\boldsymbol{X}$ and $\boldsymbol{Y}_{\boldsymbol{f}}$ for all $f$, the stimulation frequency with the maximal canonical correlation value can be identified, which is selected as the estimated SSVEP frequency.

One of the main advantages of using CCA in SSVEP-BCIs is that it can be used without any training data. In order to retain these benefits, this study is focused on normalization methods that can classify commands without the use of training data. For convenience, CCA without any normalization methods applied will be referred to as Standard CCA from this point onwards. As noted in Section I, SSVEPs elicited by higher frequency RVS are harder to detect. As such, two new methods aimed at improving the accuracy of Standard CCA through the use of pre-trial normalization have been proposed.

\section{E. Proposed Methods: Pre-Trial Normalization}

Three CCA methods are compared in this paper; they are described below along with plots of their correlation values across time for a single participant (values are averaged across 30 trials).

Standard CCA: This is CCA without any normalization method applied (Fig. 4)

Baseline-Corrected CCA: BC-CCA subtracts baseline correlation values from the Standard CCA correlation scores at the target time (Fig. 5);

Scaled CCA: This method divides the Standard CCA correlation scores at the target time by the baseline correlation values (Fig. 6).

The first step of applying either normalization method requires data from the pre-trial fixation period, during which no RVS is displayed on-screen (Fig. 3). A baseline correlation score is calculated across the pre-fixation period by calculating the maximum canonical correlation for each class multiple times using an overlapping window. Taking the mean of these scores gives a single value for each class, which will be termed the "baseline $\rho$ " for convenience. This can be calculated for each class using:

$$
\text { baseline } \rho=\frac{1}{K} \sum_{i=1}^{K} \rho\left(\boldsymbol{w}_{\boldsymbol{i}}, \boldsymbol{Y}_{\boldsymbol{f}}\right)
$$

where $\mathrm{K}$ is the total number of time windows used, and $\boldsymbol{w}_{i}$ represents the $i$ th time window of data.

Later in the trial, the baseline $\rho$ can be used to perform normalization against the Standard CCA correlation scores using either BC-CCA:

$$
B C C C A=\rho\left(\boldsymbol{x}, \boldsymbol{Y}_{\boldsymbol{f}}\right)-\text { baseline } \rho
$$

or Scaled CCA:

$$
\text { scaled } C C A=\frac{\rho\left(\boldsymbol{x}, \boldsymbol{Y}_{\boldsymbol{f}}\right)}{\text { baseline } \rho}
$$

with the maximum value across classes selected as the classifier output.

\section{F. Method Application}

Normalization requires calculating the correlation coefficients for each class several times during a single trial. To achieve this, the EEG data was downsampled to 250 $\mathrm{Hz}$ and separated into analysis windows using MATLAB plugin FieldTrip [31]. Each analysis window contained one second of data, filtered from 1-49 Hz using a zero-phase Butterworth band-pass filter with two seconds of data padding on either side. The $\rho$ values of each class were calculated for every analysis window. The start points of the analysis windows, that is, the left corners, were positioned as follows: the windows for calculating baseline $\rho$ were offset to $t \Delta=[-2,-1.8,-1.6,-1.4,-1.2]$ seconds, relative to $t 0$ (stimulus onset). These overlapping one-second windows 


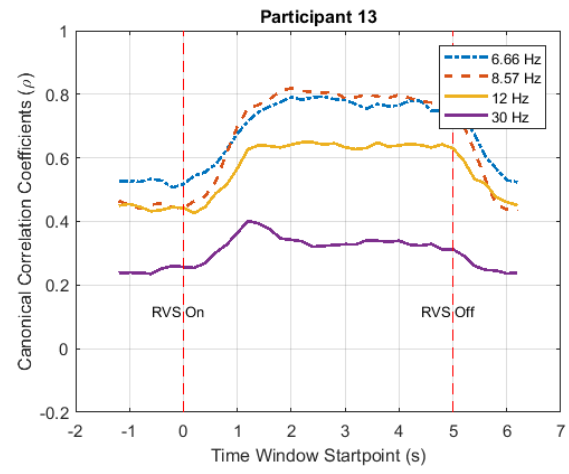

Fig. 4. Standard canonical correlation coefficients

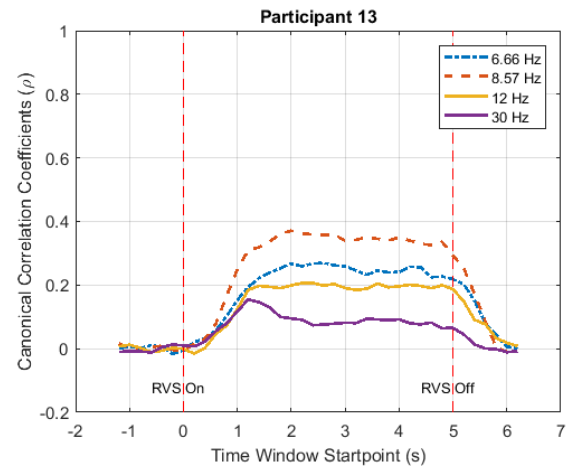

Fig. 5. Baseline-corrected canonical correlation coefficients

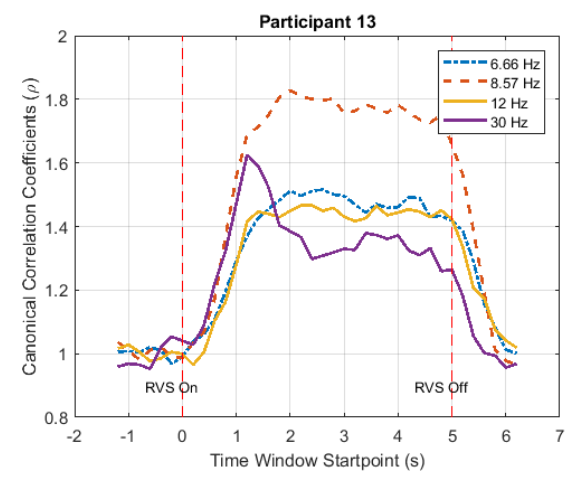

Fig. 6. Scaled canonical correlation coefficients

effectively covered most of the two-second period between the previous trial and stimulus onset of the current trial. The analysis windows for calculating Standard CCA was offset to $t \Delta=1$ second relative to $t 0$, in order to avoid the "dead time" [32], the period occurring after RVS onset but before the SSVEP response reaches maximum effectiveness.

Offline analysis tests were conducted using four different frequencies at a time, as four commands can provide enough degrees of freedom to control many simple games or assistive devices. As mentioned in section III-B, data was collected from eight RVS frequencies. However, frequencies that are multiples of one another can cause inter-harmonic interference; for example a $10 \mathrm{~Hz}$ RVS can elicit a $20 \mathrm{~Hz}$ SSVEP response. As a result, these frequencies should not be used together in a BCI. To circumvent this problem, the eight available
TABLE I. FREQUENCY COMBINATION GROUPS

\begin{tabular}{|c|c|c|c|c|c|}
\hline Condition & Freq. 1 & Freq. 2 & Freq. 3 & Freq. 4 & Range \\
\hline \hline Low & $6.66 \mathrm{~Hz}$ & $7.5 \mathrm{~Hz}$ & $8.57 \mathrm{~Hz}$ & $12 \mathrm{~Hz}$ & $5.34 \mathrm{~Hz}$ \\
Medium & $8.57 \mathrm{~Hz}$ & $12 \mathrm{~Hz}$ & $15 \mathrm{~Hz}$ & $20 \mathrm{~Hz}$ & $11.43 \mathrm{~Hz}$ \\
Wide Range & $6.66 \mathrm{~Hz}$ & $8.57 \mathrm{~Hz}$ & $12 \mathrm{~Hz}$ & $30 \mathrm{~Hz}$ & $23.43 \mathrm{~Hz}$ \\
\hline
\end{tabular}

TABLE II. MEAN ACCURACY ACROSS CONDITIONS

\begin{tabular}{|c|c|c|c|}
\hline Condition & Standard CCA (\%) & BC-CCA (\%) & Scaled CCA (\%) \\
\hline \hline Low & $\mathbf{7 3 . 4 8}$ & 72.99 & 71.13 \\
Medium & 64.46 & $\mathbf{7 1 . 7 2}$ & 70.20 \\
Wide Range & 57.84 & $\mathbf{6 7 . 0 6}$ & 64.41 \\
\hline
\end{tabular}

stimulus frequencies were separated into three conditions: Low Frequency, Medium Frequency, and Wide Range conditions (Table I). These three conditions allowed for combinations of RVS frequencies contained the lowest possible, the highest possible, and the widest range of RVS frequencies possible that had no inter-frequency interference within the first three harmonics. The $10 \mathrm{~Hz}$ RVS was not included in any condition, as there were only two RVS frequencies $(8.57$ and $12 \mathrm{~Hz}$ ) with which it shared no harmonics within this range. Analysis included all 30 trials for each class, giving a total of 120 trials per condition. Each trial had Standard CCA, Scaled CCA, and BC-CCA applied to it.

\section{RESUlTS}

Each participant had their data (120 trials per condition, three conditions) analysed using the Standard CCA, Scaled CCA, and BC-CCA methods (Fig. 7). The highest accuracies were found in the Low Frequency condition (mean = $72.53 \%$ ), followed by the Medium Frequency condition (mean $=68.79 \%$ ), with the lowest accuracies found in the Wide Range condition $($ mean $=63.11 \%$ ). Standard CCA has a very slightly improved performance in the Low Frequency condition (+0.49\%); however, both Scaled CCA and BC-CCA outperformed it in the other conditions (Table II), with BCCCA outperforming it by $7.26 \%$ in the Medium Frequency condition, and by $9.22 \%$ in the Wide Range condition. A closer look at the Wide Range condition (Table III) shows that this effect is fairly consistent across participants, with only one user performing better using Standard CCA. Separating participants into performance-based groups using Tan et al.'s [33] threshold for acceptable BCI control accuracy $(>70 \%$ accuracy) produces 11 higher accuracy participants versus 6 lower accuracy participants. The performance of these groups in the Wide Range condition suggests that the majority of improvements are made by the more accurate participants (Fig. $8,+11.81 \%$ ), with less change attributed to the less accurate participants (Fig. 9, $+4.45 \%$ ). Fig. 8 shows that the normalization techniques improved the performance of the more accurate participants, with the lower quartile of the normalized box plots almost equalling the upper quartile of the standard CCA box plot. It also shows an increased range in accuracy between the minimum and maximum scores when using normalization. Fig. 9 shows that normalization made little difference in the less accurate participants. 


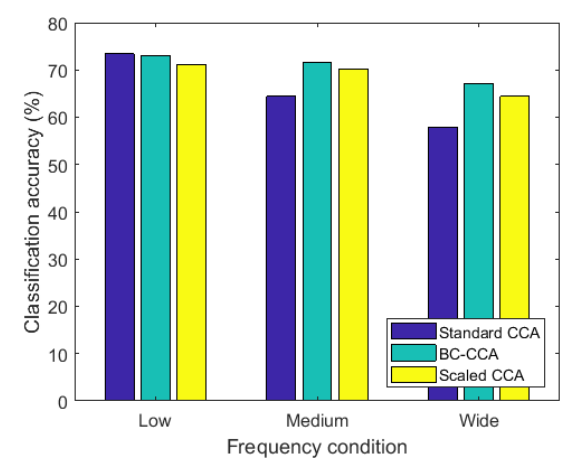

Fig. 7. Mean classification accuracy

TABLE III. CLASSIFICATION ACCURACY (WIDE RANGE CONDITION)

\begin{tabular}{|c|c|c|c|}
\hline Participant & Standard CCA (\%) & BC-CCA $(\%)$ & Scaled-CCA (\%) \\
\hline \hline 1 & 33.33 & $\mathbf{3 4 . 1 7}$ & 33.33 \\
2 & 75.83 & 87.50 & $\mathbf{8 9 . 1 7}$ \\
3 & 50 & $\mathbf{5 0 . 8 3}$ & 49.17 \\
4 & 75 & $\mathbf{8 7 . 5 0}$ & 83.33 \\
5 & 35 & 40 & $\mathbf{4 4 . 1 7}$ \\
6 & 61.67 & $\mathbf{7 0 . 8 3}$ & $\mathbf{7 0 . 8 3}$ \\
7 & 76.67 & $\mathbf{9 6 . 6 7}$ & 90 \\
8 & 50.83 & $\mathbf{5 7 . 5 0}$ & 51.67 \\
9 & 55.83 & 64.17 & $\mathbf{6 6 . 6 7}$ \\
10 & 35.83 & 41.67 & $\mathbf{4 2 . 5 0}$ \\
11 & 26.67 & $\mathbf{3 4 . 1 7}$ & 30.83 \\
12 & 61.67 & $\mathbf{7 4 . 1 7}$ & $\mathbf{7 4 . 1 7}$ \\
13 & 72.50 & 85.83 & $\mathbf{8 6 . 6 7}$ \\
14 & 70 & $\mathbf{8 7 . 5 0}$ & 80.83 \\
15 & 74.17 & $\mathbf{9 4 . 1 7}$ & 85 \\
16 & 70 & $\mathbf{8 0}$ & 70 \\
17 & $\mathbf{5 8 . 3 3}$ & 53.33 & 46.67 \\
\hline Mean & 57.84 & 67.06 & 64.41 \\
\hline
\end{tabular}

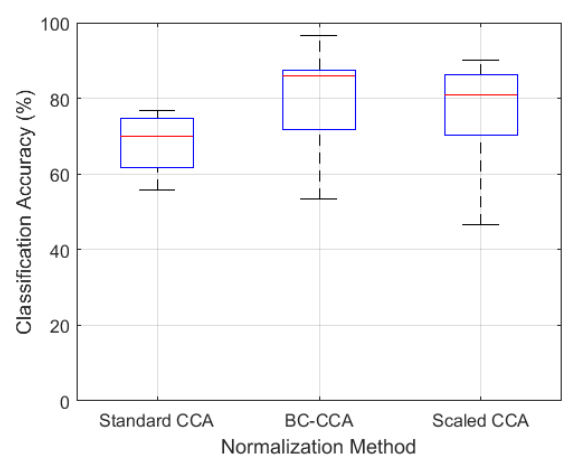

Fig. 8. Higher accuracy participants (wide condition)

\section{Discussion}

This study has investigated the problem of whether it is possible to further improve CCA performance without the use of training data, through the application of pre-trial normalization methods. The results show that it is indeed possible, and its effectiveness is dependent upon the RVS frequencies selected for use. Both Scaled CCA and BC-CCA were found to be effective in some cases: for low frequencies BC-CCA $(-0.49 \%)$ and Scaled CCA $(-2.35 \%)$ were outperformed by Standard

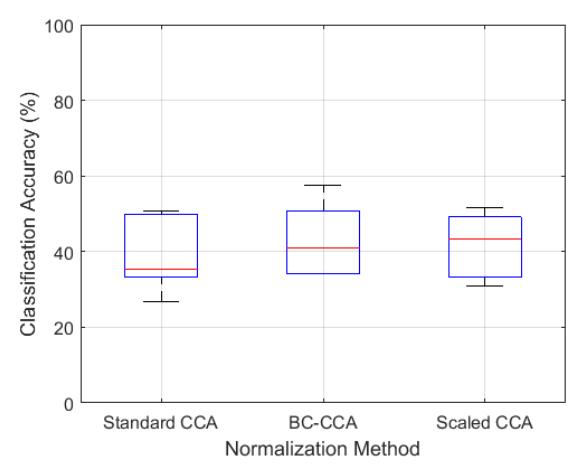

Fig. 9. Lower accuracy participants (wide condition)

CCA; however, for medium frequencies, BC-CCA $(+7.26 \%)$ and Scaled CCA $(+5.74 \%)$ both had a higher accuracy than Standard CCA, and both outperformed it again for wide range frequencies $(\mathrm{BC}-\mathrm{CCA}=+9.22 \%$, Scaled $\mathrm{CCA}=+6.57 \%)$. The results support both the hypotheses that SSVEP-BCI performance can be improved using CCA data from the prefixation period, and that trials from wide-ranged frequencies would have the greatest improvement. As expected Standard CCA classification accuracy deteriorates as the range between RVS frequencies increases, while it stays fairly consistent when normalization methods are applied.

As shown by the plots of each method's canonical coefficient values across time (Figs. 4, 5, and 6), BC-CCA and Scaled CCA appear to minimize the difference between the CCA coefficients, thereby making it more likely that weaker SSVEP responses such as at 20 and $30 \mathrm{~Hz}$ can be correctly detected. However, it is unclear why BC-CCA appears to perform better than Scaled CCA on a fairly consistent basis. As it is a baseline correction method, BC-CCA preserves the changes of each frequencies correlation score over time, relative to itself; it simply equalizes their value at t0. Whereas, Scaled CCA effectively applies a penalty to frequencies with a high baseline $\rho$, and applies that to their correlation score at every time point which should theoretically allow weaker frequencies a stronger response. This should give some insight into why the methods perform differently, although further work is required to determine which situations are preferable for each method.

Future work should look at whether training data can be used to further improve the results of BC-CCA and Scaled $\mathrm{CCA}$, and test their effectiveness with a larger number of frequencies. A more structured approach to selecting the pretrial fixation period may reduce the computations required for real-time control.

\section{CONCLUSION}

BC-CCA and Scaled CCA were both found to be effective normalization methods, mitigating the decrease in BCI performance seen as the distance between frequencies increases, thus allowing a greater range of visual stimulus frequencies to be selected. Of all the methods investigated, BC-CCA was found to be the most effective. 


\section{ACKNOWLEDGEMENT}

The authors would like to thank Sheffield Robotics for funding this research, and would also like to thank the anonymous referees for their helpful comments.

\section{REFERENCES}

[1] G. Pfurtscheller, D. Flotzinger, and J. Kalcher, "BrainComputer Interface-a new communication device for handicapped persons," pp. 293-299, 1993. [Online]. Available: http://dx.doi.org/10.1006/jmca.1993.1030

[2] R. P. N. Rao, Brain-Computer Interfacing: An Introduction. Cambridge University Press, 2013.

[3] P. Baranyi and A. Csapo, "Cognitive infocommunications: coginfocom," in Computational Intelligence and Informatics (CINTI), 2010 11th International Symposium on. IEEE, 2010, pp. 141-146.

[4] J. Katona, T. Ujbanyi, G. Sziladi, and A. Kovari, "Speed control of Festo Robotino mobile robot using NeuroSky MindWave EEG headset based brain-computer interface," 7th IEEE International Conference on Cognitive Infocommunications, CogInfoCom 2016 - Proceedings, no. CogInfoCom, pp. 251-256, 2017.

[5] T. Nagya, D. Telleza, Á. Divák, E. Lógób, M. Kólesb, and B. Hámornikb, "Predicting arousal with machine learning of EEG signals," 5th IEEE International Conference on Cognitive Infocommunications, CogInfoCom 2014 - Proceedings, pp. 137-140, 2014.

[6] J. Henshaw, W. Liu, and D. Romano, "Problem Solving Using Hybrid Brain-Computer Interface Methods : a Review," in Cognitive Infocommunications (CogInfoCom), 2014 5th IEEE Conference on, 2014, pp. 215-219.

[7] G. R. Mcmillan, G. L. Calhoun, M. S. Middendorf, J. H. Schnurer, D. F Ingle, and V. T. Nasman, "Direct brain interface utilizing self-regulation of steady-state visual evoked response (SSVER)," Rehabilitation Engineering and Assistive Technology Society of North America, no. Figure 1, pp. 693-695, 1995.

[8] C. S. Herrmann, "Human EEG responses to 1-100 Hz flicker: Resonance phenomena in visual cortex and their potential correlation to cognitive phenomena," Experimental Brain Research, vol. 137, no. 3-4, pp. 346-353, 2001.

[9] H. Bakardjian, T. Tanaka, and A. Cichocki, "Optimization of SSVEP brain responses with application to eight-command Brain-Computer Interface." Neuroscience letters, vol. 469, no. 1, pp. 34-38, jan 2010. [Online]. Available: http://www.ncbi.nlm.nih.gov/pubmed/19931592

[10] G. R. Burkitt, R. B. Silberstein, P. J. Cadusch, and a. W. Wood, "Steady-state visual evoked potentials and travelling waves." Clinical neurophysiology : official journal of the International Federation of Clinical Neurophysiology, vol. 111, no. 2, pp. 246-258, 2000.

[11] B. Graimann, B. Allison, and G. Pfurtscheller, Brain-computer interfaces: Revolutionizing human-computer interaction. Springer Science \& Business Media, 2010.

[12] H. Gürkök, A. Nijholt, M. Poel, and M. Obbink, "Evaluating a multiplayer brain-computer interface game: Challenge versus co-experience," Entertainment Computing, vol. 4, no. 3, pp. 195-203, 2013.

[13] T. Sakurada, T. Kawase, K. Takano, T. Komatsu, and K. Kansaku, "A BMI-based occupational therapy assist suit: Asynchronous control by SSVEP," Frontiers in Neuroscience, vol. 7, no. 7 SEP, pp. 1-10, 2013.

[14] S. M. T. Müller and T. F. Bastos-filho, "Brain-computer Interface Based on Visual Evoked Potentials to Command Autonomous Robotic Wheelchair," Journal of Medical and Biological Engineering, vol. 30 no. 6, pp. 407-416, 2010.

[15] G. Vanacker, J. Del R. Millán, E. Lew, P. W. Ferrez, F. G. Moles, J. Philips, H. Van Brussel, and M. Nuttin, "Context-based filtering for assisted brain-actuated wheelchair driving," Computational Intelligence and Neuroscience, vol. 2007, pp. 1-12, 2007.

[16] C. J. Bell, P. Shenoy, R. Chalodhorn, and R. P. N. Rao, "Control of a humanoid robot by a noninvasive brain-computer interface in humans.' Journal of neural engineering, vol. 5, no. 2, pp. 214-20, jun 2008 [Online]. Available: http://www.ncbi.nlm.nih.gov/pubmed/18483450
[17] Z. Lin, C. Zhang, W. Wu, and X. Gao, "Frequency Recognition Based on Canonical Correlation Analysis for SSVEP-Based BCIs," IEEE Transactions on Biomedical Engineering, vol. 53, no. 12, pp. 315-323, 2006. [Online]. Available: http://link.springer.com/10.1007/978-3-31912436-0

[18] G. Bin, X. Gao, Z. Yan, B. Hong, and S. Gao, "An online multi-channel SSVEP-based brain-computer interface using a canonical correlation analysis method." Journal of neural engineering, vol. 6, no. 4, p. 046002, 2009

[19] M. Nakanishi, Y. Wang, Y.-t. Wang, Y. Mitsukura, and T. Jung, "Enhancing Unsupervised Canonical Correlation Analysis-Based Frequency Detection of SSVEPs by Incorporating Background EEG," in 36th Annual International Conference of the IEEE Engineering in Medicince and Biology Society, 2014, pp. 3053-3056.

[20] Y. Zhang, G. Zhou, J. Jin, X. Wang, and A. Cichocki, "FREQUENCY RECOGNITION IN SSVEP-BASED BCI USING MULTISET CANONICAL CORRELATION ANALYSIS," International Journal of Neural Systems, vol. 24, no. 4, p. 1450013, 2014.

[21] S. N. Carvalho, T. B. Costa, L. F. Uribe, D. C. Soriano, G. F. Yared, L. C. Coradine, and R. Attux, "Comparative analysis of strategies for feature extraction and classification in SSVEP BCIs," Biomedical Signal Processing and Control, vol. 21, pp. 34-42, 2015. [Online]. Available: http://linkinghub.elsevier.com/retrieve/pii/S1746809415000877

[22] J. Castillo, S. Muller, E. Caicedo, and T. Bastos, "Feature extraction techniques based on power spectrum for a SSVEP-BCI," in 2014 IEEE 23rd International Symposium on Industrial Electronics (ISIE), 2014, pp. 1051-1055. [Online]. Available: http://ieeexplore.ieee.org/lpdocs/epic03/wrapper.htm?arnumber=6864758

[23] S. Resalat and S. Setarehdan, "An Improved SSVEP Based BCI System Using Frequency Domain Feature Classification," American Journal of Biomedical ..., vol. 3, no. 1, pp. 1-8, 2013. [Online]. Available: http://article.sapub.org/10.5923.j.ajbe.20130301.01.html

[24] P. Yuan, X. Gao, B. Allison, Y. Wang, G. Bin, and S. Gao, "A study of the existing problems of estimating the information transfer rate in online brain-computer interfaces." Journal of neural engineering, vol. 10, no. 2, p. 026014, 2013. [Online]. Available: http://www.ncbi.nlm.nih.gov/pubmed/23448963

[25] T. Sakurada, T. Kawase, T. Komatsu, and K. Kansaku, "Use of high-frequency visual stimuli above the critical flicker frequency in a SSVEP-based BMI," Clinical Neurophysiology, vol. 126, no. 10, pp. 1972-1978, 2015. [Online]. Available: http://dx.doi.org/10.1016/j.clinph.2014.12.010

[26] P. F. Diez, V. A. Mut, E. Laciar, and E. M. A. Perona, "Mobile robot navigation with a self-paced braincomputer interface based on high- frequency SSVEP," Robotica, vol. 32, no. November 2013, pp. 695-709, 2014. [Online]. Available: http://www.scopus.com/inward/record.url?eid=2s2.0-84888194023\&partnerID=tZOtx3y1

[27] D. H. Brainard, "The Psychophysics Toolbox.” Spatial vision, vol. 10, no. 4, pp. 433-436, 1997.

[28] D. G. Pelli, "The VideoToolbox software for visual psychophysics: transforming numbers into movies." pp. 437-442, 1997.

[29] M. Kleiner, D. Brainard, D. Pelli, A. Ingling, R. Murray, and C. Broussard, "What's new in Psychtoolbox-3 ? Foreword ; - )," Perception, vol. 36 , no. 14, pp. 1-89, 2007.

[30] H. Cecotti, I. Volosyak, and A. Gräser, "RELIABLE VISUAL STIMULI ON LCD SCREENS FOR SSVEP BASED BCI," in The 2010 European Signal Processing Conference (EUSIPCO-2010), 2010, pp. 919-923.

[31] R. Oostenveld, P. Fries, E. Maris, and J. M. Schoffelen, "FieldTrip: Open source software for advanced analysis of MEG, EEG, and invasive electrophysiological data," Computational Intelligence and Neuroscience, vol. 2011, 2011.

[32] M. Sengelmann, A. K. Engel, and A. Maye, "Maximizing information transfer in SSVEP-based brain computer interfaces," IEEE Transactions on Biomedical Engineering, vol. 9294, no. c, pp. 1-1, 2016. [Online]. Available: http://ieeexplore.ieee.org/lpdocs/epic03/wrapper.htm?arnumber=7460915

[33] D. Tan and A. Nijholt, Human-computer Interaction, 2010. 